\title{
ON THE SIGNATURE OF CERTAIN INTERSECTION FORMS
}

\author{
FENG XU
}

\begin{abstract}
We prove a conjecture of Zuber on the signature of intersection froms associated with affine algebras of type A.
\end{abstract}

\section{$\S 1$. INTRODUCTION}

Let $N \geq 2$ be a positive integer and $\lambda^{\prime}=\left(\lambda_{1}^{\prime}, \ldots, \lambda_{N-1}^{\prime}\right), 0<\lambda_{i}^{\prime}<1, i=$ $1,2, \ldots, N-1$ with $\sum_{0<i<N} \lambda_{i}^{\prime}<1$. Define

$$
p_{i}\left(\lambda^{\prime}\right):=\lambda_{i}^{\prime}+\ldots+\lambda_{N-1}^{\prime}-\frac{1}{N} \sum_{0<j<N} j \lambda_{j}^{\prime}, i=1,2, \ldots, N-1
$$

and $p_{N}\left(\lambda^{\prime}\right):=-\frac{1}{N} \sum_{0<j<N} j \lambda_{j}^{\prime}$. Define

$$
q_{i}\left(\lambda^{\prime}\right):=-N p_{i}\left(\lambda^{\prime}\right)+\frac{N+1}{2}-i
$$

and

$$
g_{i}\left(\lambda^{\prime}\right):=(-1)^{i} \prod_{r=1}^{N} 2 \cos \left(\pi\left(p_{r}\left(\lambda^{\prime}\right)-\frac{i}{N}\right)\right)
$$

$, i=1,2, \ldots, N$.

If $S$ is a finite sequence of real numbers, we define $b_{+}(S)\left(\operatorname{resp} . b_{-}(S), b_{0}(S)\right)$ to be the number of positive (resp. negative, zero) elements in $S$. Let $a(S):=$ $b_{+}(S)-b_{-}(S)$ and denote by $Q_{\lambda^{\prime}}, G_{\lambda^{\prime}}$ the following two sets:

$$
\begin{gathered}
Q_{\lambda^{\prime}}:=\left\{\cos \left(\pi q_{1}\left(\lambda^{\prime}\right)\right), \ldots, \cos \left(\pi q_{N}\left(\lambda^{\prime}\right)\right)\right\}, \\
G_{\lambda^{\prime}}:=\left\{g_{1}\left(\lambda^{\prime}\right), \ldots, g_{N}\left(\lambda^{\prime}\right)\right\} .
\end{gathered}
$$

Notice that since $\cos \left(\pi q_{i}\left(\lambda^{\prime}\right)\right)>0$ iff $\left.q_{i}\left(\lambda^{\prime}\right) \in\right] 2 p-\frac{1}{2}, 2 p+\frac{1}{2}$ [ for some integer $p$, $b_{+}\left(Q_{\lambda^{\prime}}\right)$ is much easier to calculate than $b_{+}\left(G_{\lambda^{\prime}}\right)$ and the same is true for $b_{-}$'s. The main theorem in this paper is the following:

This work is partially supported by NSF grant DMS-9500882.

Typeset by $\mathcal{A} \mathcal{M} \mathcal{S}-\mathrm{T}_{\mathrm{E}} \mathrm{X}$ 
Theorem 1. Let $\lambda^{\prime}=\left(\lambda_{1}^{\prime}, \ldots, \lambda_{N-1}^{\prime}\right)$ be as above. Then:

$$
b_{+}\left(Q_{\lambda^{\prime}}\right)=b_{+}\left(G_{\lambda^{\prime}}\right), b_{-}\left(Q_{\lambda^{\prime}}\right)=b_{-}\left(G_{\lambda^{\prime}}\right), b_{0}\left(Q_{\lambda^{\prime}}\right)=b_{0}\left(G_{\lambda^{\prime}}\right)
$$

This theorem implies Zuber's conjecture about the signature of intersection forms associated with affine algebras of type A (cf.[Z1]) which is the motivation of this paper. Note that $b_{0}\left(Q_{\lambda^{\prime}}\right)=b_{0}\left(G_{\lambda^{\prime}}\right)$ is already noticed in a slightly different form in $[\mathrm{Z} 1]$.

Zuber's conjecture appeared as Conjecture 2.5 of [Z1]. It is based on the mysterious connections between integrable models with two supersymmetries $(\mathrm{N}=2)$ in two dimensions (cf.[CV]) and the class of graphs constructed in [Z1] (see also [Z2]). In the special case when the graphs are regular (cf.§2.3), the conjecture can be proved (cf. Page 14 of [GZV]) by combining the results of [GZV] and [S]. In fact, in [GZV], a connection between regular graphs and singularity theory is established, and combine with [S] which is based on mixed Hodge structures, gives a rather indirect proof of Zuber's conjecture in the special case when the graphs are regular. However, for other graphs in [Z1] (see also $[\mathrm{X}]$ ), the connection with singularity theory, if any, is not clear at all. We also failed to prove Zuber's conjecture by using the connection with [CV] as mentioned in [Z1].

We came to the realization that a statement as in theorem 1 may be true by first observing lemma 1 (cf.§2.1) which was already noticed in a slightly different form in [Z1]. We then checked that theorem 1 is true explicitly in the case when $N=3,4$ and some other cases which motivated us to give a general proof.

The idea of the proof of theorem 1 is as follows. When $\lambda^{\prime}$ changes, $b_{+}\left(G_{\lambda^{\prime}}\right)$ (resp. $b_{-}\left(G_{\lambda^{\prime}}\right)$ ) may change only if some of $g_{i}\left(\lambda^{\prime}\right)$ 's become 0 or change its sign, i.e., $g_{i}\left(\lambda^{\prime}\right)$ intersect the hyperplanes on which $g_{i}\left(\lambda^{\prime}\right)=0$. By lemma 1 of $\S 2.1$, these hyperplanes are the same as the hyperplanes on which some $q_{j}\left(\lambda^{\prime}\right)$ 's lie in $\mathbb{Z}+\frac{1}{2}$. Consider the domain $D:=\left\{\lambda^{\prime}=\left(\lambda_{1}^{\prime}, \ldots \lambda_{N-1}^{\prime}\right) \mid 0<\lambda_{i}^{\prime}<1, \sum_{0<i<N} \lambda_{i}^{\prime}<1\right\}$. $D$ is separated by the above hyperplanes into disjoint open regions. In each open region, the set of numbers compared in theorem 1 should be completely determined. In $\S 2.1$, we determine these numbers in a given open region and find that they miraculously satisfy theorem 1 . In $\S 2.2$, we show that theorem 1 also holds for any $\lambda^{\prime} \in D$ which is on the boundary of the open region: this follows from $\S 2.1$ and lemma 1 . In $\S 2.3$, after introducing Zuber's conjecture, we show how theorem 1 implies that the conjecture is true. In $\S 3$, we present our conclusions and questions.

\section{THE PROOF}

We shall use the notations of $\S 1$. Recall

$$
\begin{aligned}
D=\left\{\lambda^{\prime}=\right. & \left.\left(\lambda_{1}^{\prime}, \ldots, \lambda_{N-1}^{\prime}\right) \mid \lambda_{i}^{\prime}>0, \sum_{0<i<N} \lambda_{i}^{\prime}<1\right\} . \text { For } \lambda^{\prime} \in D, \text { recall } \\
p_{i}\left(\lambda^{\prime}\right) & =\lambda_{i}^{\prime}+\ldots+\lambda_{N-1}^{\prime}-\frac{1}{N} \sum_{0<j<N} j \lambda_{j}^{\prime} \\
& =\frac{1}{N}\left[\left(-\lambda_{1}^{\prime}-2 \lambda_{2}^{\prime}-\ldots-(i-1) \lambda_{i-1}^{\prime}\right)+(N-i) \lambda_{i}^{\prime}+\ldots+\lambda_{N-1}^{\prime}\right]
\end{aligned}
$$


for $i=1,2, \ldots, N-1$. We have:

$$
\begin{aligned}
p_{i}\left(\lambda^{\prime}\right) & >\frac{1}{N}\left[\left(-\lambda_{1}^{\prime}-2 \lambda_{2}^{\prime}-\ldots-(i-1) \lambda_{i-1}^{\prime}\right)\right] \\
& >-\frac{1}{N}(i-1) \sum_{0<j<N} \lambda_{j}^{\prime}>-\frac{1}{N}(i-1)
\end{aligned}
$$

, and

$$
\begin{aligned}
p_{i}\left(\lambda^{\prime}\right) & <\frac{1}{N}\left[(N-i) \lambda_{i}^{\prime}+\ldots+\lambda_{N-1}^{\prime}\right] \\
& <\frac{1}{N}(N-i) \sum_{0<j<N} \lambda_{j}^{\prime}<\frac{1}{N}(N-i)
\end{aligned}
$$

. Similarly one can show $\frac{1}{N}(1-N)<p_{N}\left(\lambda^{\prime}\right)<0$. So we have:

$$
\frac{1}{N}(1-i)<p_{i}\left(\lambda^{\prime}\right)<\frac{1}{N}(N-i)
$$

$, i=1,2, \ldots, N$. If for some $i, q_{i}\left(\lambda^{\prime}\right)=-N p_{i}\left(\lambda^{\prime}\right)+\frac{1}{2}(N+1)-i=j+\frac{1}{2}$ with $j \in \mathbb{Z}$, then

$$
i-\frac{1}{2} N<i+j<i+\frac{1}{2} N-1
$$

. Define $0<r_{\lambda^{\prime}}(i)<N+1$ to be the unique integer such that $\frac{1}{N}\left(r_{\lambda^{\prime}}(i)+j+i\right) \in \mathbb{Z}$. In fact, if $j+i<0, r_{\lambda^{\prime}}(i)=-(j+i)$, if $0 \leq j+i<N, r_{\lambda^{\prime}}(i)=N-(j+i)$, and if $N \leq(j+i)<\frac{3}{2} N, r_{\lambda^{\prime}}(i)=2 N-(j+i)$. It follows that

$$
g_{r_{\lambda^{\prime}}(i)}\left(\lambda^{\prime}\right)=0
$$

- We have the following lemma which, in a slightly different form also appeared on Page 17 of [Z1].

lemma 1. For any $\lambda^{\prime} \in D$, the map $i \rightarrow r_{\lambda^{\prime}}(i)$ defined above from $\left\{i \mid q_{i}\left(\lambda^{\prime}\right) \in \mathbb{Z}+\frac{1}{2}\right\}$ to $\left\{r \mid g_{r}\left(\lambda^{\prime}\right)=0\right\}$ is a one to one and onto map. Moreover $q_{i}\left(\lambda^{\prime}\right) \in \mathbb{Z}+\frac{1}{2}$ iff $g_{r_{\lambda^{\prime}}(i)}\left(\lambda^{\prime}\right)=0$ and $r_{\lambda^{\prime}}(i)$ depends only on $i$ and $q_{i}\left(\lambda^{\prime}\right)$.

Proof. By using definitions we have

$$
g_{r}\left(\lambda^{\prime}\right)=(-1)^{r} \prod_{i=1}^{N} 2 \sin \left(\frac{\pi}{N}\left(q_{i}\left(\lambda^{\prime}\right)+i+r-\frac{1}{2}\right)\right)
$$

. If $g_{r}\left(\lambda^{\prime}\right)=0$, then there exists $i:=i_{\lambda^{\prime}}(r)$ such that

$$
\frac{1}{N}\left(q_{i}\left(\lambda^{\prime}\right)+i+r-\frac{1}{2}\right) \in \mathbb{Z}
$$


. Let $j \in \mathbb{Z}$ with $q_{i}\left(\lambda^{\prime}\right)+i+r-\frac{1}{2}=j+i+r$, then $q_{i}\left(\lambda^{\prime}\right)=-N p_{i}\left(\lambda^{\prime}\right)+\frac{1}{2}(N+1)-i \in$ $\mathbb{Z}+\frac{1}{2}$. Using the fact that $0<\left|p_{a}-p_{b}\right|<1$ for any $1 \leq a \neq b \leq N$, it is easy to see that such an $i:=i_{\lambda^{\prime}}(r)$ is also unique. It is then easy to check that the map $i \rightarrow r_{\lambda^{\prime}}(i)$ and $r \rightarrow i_{\lambda^{\prime}}(r)$ are inverse to each other. The rest of the lemma follows from the definitions of $r_{\lambda^{\prime}}(i)$.

Q.E.D.

Let $\beta_{i}:=\frac{1}{2} N-i+\gamma_{i}$ with $\gamma_{i} \in \mathbb{Z}, i=1,2, \ldots, N$. Let $D_{\gamma}:=\left\{\lambda^{\prime} \in D \mid \beta_{i}<\right.$ $\left.N p_{i}\left(\lambda^{\prime}\right)<\beta_{i}+1, i=1,2, \ldots, N\right\} . D_{\gamma}$ will be called open regions. It is clear that if $\gamma \neq \gamma^{\prime}$, then $D_{\gamma} \cap D_{\gamma^{\prime}}=\emptyset$. Notice that $q_{i}\left(\lambda^{\prime}\right) \in \mathbb{Z}+\frac{1}{2}$ iff $\lambda^{\prime}$ lies on the boundary of some $D_{\gamma}$, and by lemma $1, g_{r_{\lambda^{\prime}}(i)}\left(\lambda^{\prime}\right)=0$ iff $\lambda^{\prime}$ lies on the boundary of some $D_{\gamma}$. Suppose $D_{\gamma} \neq \emptyset$ and $\lambda^{\prime} \in D_{\gamma}$. Then we have:

(1) If $i<j$, then $\beta_{i} \geq \beta_{j}$ which follows from the fact that $i<j$, then $p_{i}\left(\lambda^{\prime}\right)>$ $p_{j}\left(\lambda^{\prime}\right)$ and $\beta_{i}-\beta_{j} \in \mathbb{Z}$

(2) $\beta_{1}-\beta_{N} \leq N$ which follows from $p_{1}\left(\lambda^{\prime}\right)-p_{N}\left(\lambda^{\prime}\right)<1$.

By (1) we can assume that

$$
\beta_{1}=\ldots=\beta_{i_{1}}>\beta_{i_{1}+1}=\ldots=\beta_{i_{2}}>\ldots>\beta_{i_{t-1}+1}=\ldots=\beta_{i_{t}}
$$

where $1 \leq i_{1}<i_{2}<\ldots<i_{t}=N$. We determine the sign of $g_{r}\left(\lambda^{\prime}\right)$ for a fixed $1 \leq r \leq N$. Since $0<p_{1}\left(\lambda^{\prime}\right)-p_{N}\left(\lambda^{\prime}\right)<1$, there is at most one $k+\frac{1}{2}$ with $k \in \mathbb{Z}$ such that

$$
p_{N}\left(\lambda^{\prime}\right)-\frac{r}{N}<k+\frac{1}{2}<p_{1}\left(\lambda^{\prime}\right)-\frac{r}{N}
$$

. Also notice that if

$$
\frac{\beta_{i}-r}{N}<k+\frac{1}{2}<\frac{\beta_{i}-r+1}{N}
$$

, then we have

$$
\gamma_{i}-i-N k<r<\gamma_{i}-i-N k+1
$$

which is impossible since $r, \gamma_{i}$ are integers. So if there is a $k \in \mathbb{Z}$ such that

$$
p_{N}\left(\lambda^{\prime}\right)-\frac{r}{N}<k+\frac{1}{2}<p_{1}\left(\lambda^{\prime}\right)-\frac{r}{N}
$$

, then there is a unique integer, denoted by $1 \leq f(r) \leq t-1$, such that:

$$
\frac{\beta_{i_{f(r)+1}}+1-r}{N} \leq k+\frac{1}{2} \leq \frac{\beta_{i_{f(r)}}-r}{N}
$$

, and the sign of $g_{r}\left(\lambda^{\prime}\right)$ is:

$$
(-1)^{r}(-1)^{k k^{\prime}}(-1)^{(k-1)\left(N-k^{\prime}\right)}=(-1)^{r+N k-i_{f(r)}}
$$

where $k^{\prime}:=\left\{\beta_{j}: \beta_{j}<\beta_{f(r)}\right\}^{\sharp}=N-i_{f(r)}$. If there is no $k_{1} \in \mathbb{Z}$ such that

$$
p_{N}\left(\lambda^{\prime}\right)-\frac{r}{N}<k_{1}+\frac{1}{2}<p_{1}\left(\lambda^{\prime}\right)-\frac{r}{N}
$$


, then there is a $k \in \mathbb{Z}$ such that:

$$
k-\frac{1}{2} \leq \frac{\beta_{N}-r}{N}<\frac{\beta_{1}+1-r}{N} \leq k+\frac{1}{2}
$$

, and the sign of $g_{r}\left(\lambda^{\prime}\right)$ is:

$$
(-1)^{r+k N}
$$

. We define $f(r)=t$ in this case. Let $s:=r+k N$, then the signs of the set $\left\{g_{r}\left(\lambda^{\prime}\right)\right\}$ with $1 \leq f(r) \leq t-1$ are given by

$$
(-1)^{s-i_{f(r)}}
$$

with $\gamma_{i_{f(r)+1}}+1-i_{f(r)+1} \leq s \leq \gamma_{i_{f(r)}}-i_{f(r)}$, and the sign of the set $\left\{g_{r}\left(\lambda^{\prime}\right)\right\}$ with $f(r)=t$ is given by

$$
(-1)^{s-i_{t}}
$$

with $\gamma_{i_{1}}+1-i_{1}-N \leq s \leq \gamma_{i_{t}}-i_{t}$. Now we determine the sign of $\cos \left(\pi q_{i}\left(\lambda^{\prime}\right)\right)$. Recall $\beta_{i}=\frac{N}{2}-i+\gamma_{i}, q_{i}\left(\lambda^{\prime}\right)=-N p_{i}\left(\lambda^{\prime}\right)+\frac{N+1}{2}-i$ and $\beta_{i}<N p_{i}\left(\lambda^{\prime}\right)<\beta_{i}+1$, we have

$$
-\gamma_{i}-\frac{1}{2}<q_{i}\left(\lambda^{\prime}\right)<-\gamma_{i}+\frac{1}{2}
$$

. So $\cos \left(\pi q_{i}\left(\lambda^{\prime}\right)\right)>0\left(\right.$ resp. $\left.\cos \left(\pi q_{i}\left(\lambda^{\prime}\right)\right)<0\right)$ iff $\gamma_{i} \in 2 \mathbb{Z}\left(\right.$ resp. $\left.\gamma_{i} \in 2 \mathbb{Z}+1\right)$. Recall from the introduction we have that for a finite sequence $S$ of real numbers $a(S)=$ $b_{+}(S)-b_{-}(S)$. To save some writing for any integer $x$ we define $\{x\}:=\frac{1-(-1)^{x}}{2}$. Then the $a$ of the following sequence $\left\{\cos \left(\pi q_{i}\left(\lambda^{\prime}\right)\right), i_{u-1}+1 \leq i \leq i_{u}\right\}$ is

$$
(-1)^{\gamma_{i_{u}}}\left\{i_{u}-i_{u-1}\right\}
$$

and the $a$ of the following sequence $\left\{(-1)^{s-i_{u}}, \gamma_{i_{u+1}}+1-i_{u+1} \leq s \leq \gamma_{i_{u}}-i_{u}\right\}$ is

$$
(-1)^{\gamma_{i_{u}}}\left\{\gamma_{i_{u}}-\gamma_{i_{u+1}}+i_{u+1}-i_{u}\right\}
$$

, where we define $i_{u-1}=0$ if $u=1$, and $\gamma_{i_{u+1}}-i_{u+1}=\gamma_{i_{1}}-i_{1}-N$ if $u=t$. It follows that $a\left(G_{\lambda^{\prime}}\right)-a\left(Q_{\lambda^{\prime}}\right)$ is given by

$$
\begin{aligned}
\sum_{u=1}^{t} & (-1)^{\gamma_{i_{u}}}\left(\left\{\gamma_{i_{u}}-\gamma_{i_{u+1}}+i_{u+1}-i_{u}\right\}-\left\{i_{u}-i_{u-1}\right\}\right) \\
= & (-1)^{\gamma_{i_{1}}}\left(\left\{\gamma_{i_{1}}-\gamma_{i_{2}}+i_{2}-i_{1}\right\}-\left\{i_{1}\right\}\right)+ \\
& (-1)^{\gamma_{i_{2}}}\left(\left\{\gamma_{i_{2}}-\gamma_{i_{3}}+i_{3}-i_{2}\right\}-\left\{i_{2}-i_{1}\right\}\right)+ \\
& \quad \ldots+(-1)^{\gamma_{i_{t-1}}}\left(\left\{\gamma_{i_{t-1}}-\gamma_{i_{t}}+i_{t}-i_{t-1}\right\}-\left\{i_{t-1}-i_{t-2}\right\}\right)+ \\
& (-1)^{\gamma_{i_{t}}}\left(\left\{\gamma_{i_{t}}-\gamma_{i_{1}}+i_{1}\right\}-\left\{i_{t}-i_{t-1}\right\}\right)
\end{aligned}
$$

. By using

$$
\left\{\gamma_{i_{u}}-\gamma_{i_{u+1}}+i_{u+1}-i_{u}\right\}=\left\{\gamma_{i_{u}}-\gamma_{i_{u+1}}\right\}+(-1)^{\gamma_{i_{u}}-\gamma_{i_{u+1}}}\left\{i_{u+1}-i_{u}\right\}
$$


which follows easily from the definition of $\{$.$\} , we see that \pm\left\{i_{u+1}-i_{u}\right\}$ terms cancelled each other in the above summation and the remaining terms are:

$$
(-1)^{\gamma_{i_{1}}}\left\{\gamma_{i_{1}}-\gamma_{i_{2}}\right\}+(-1)^{\gamma_{i_{2}}}\left\{\gamma_{i_{2}}-\gamma_{i_{3}}\right\}+\ldots+(-1)^{\gamma_{i_{t}}}\left\{\gamma_{i_{t}}-\gamma_{i_{1}}\right\}
$$

which is also 0 since $\{x\}=\frac{1-(-1)^{x}}{2}$. So we have shown that

$$
a\left(G_{\lambda^{\prime}}\right)-a\left(Q_{\lambda^{\prime}}\right)=0
$$

, i.e.,

$$
b_{+}\left(G_{\lambda^{\prime}}\right)-b_{-}\left(G_{\lambda^{\prime}}\right)=b_{+}\left(Q_{\lambda^{\prime}}\right)-b_{-}\left(Q_{\lambda^{\prime}}\right)
$$

. Since

$$
b_{+}\left(G_{\lambda^{\prime}}\right)+b_{-}\left(G_{\lambda^{\prime}}\right)=N=b_{+}\left(Q_{\lambda^{\prime}}\right)+b_{-}\left(Q_{\lambda^{\prime}}\right)
$$

, it follows that theorem 1 is true for $\lambda^{\prime} \in D_{\gamma}$.

2.2. The boundary case. Assume $\lambda^{\prime} \in D$ and $\lambda^{\prime}$ is on the boundary of some $D_{\gamma}$. Assume $\left\{q_{i}\left(\lambda^{\prime}\right) \mid q_{i}\left(\lambda^{\prime}\right) \in \mathbb{Z}+\frac{1}{2}\right\}=\left\{q_{k_{1}}\left(\lambda^{\prime}\right), \ldots, q_{k_{s}}\left(\lambda^{\prime}\right), s \geq 1\right\}$. Let $k_{i} \rightarrow r_{\lambda^{\prime}}\left(k_{i}\right)$ be as in lemma 1. We can choose a small neighborhood $W$ of $\lambda^{\prime}$ such that for any $\mu \in W$ and $l \neq k_{i}, i=1, \ldots, s$ (resp. $\left.m \neq r\left(k_{i}\right), i=1, \ldots, s\right), \cos \left(\pi q_{l}(\mu)\right)$ (resp. $\left.g_{m}(\mu)\right)$ has the same sign as $\cos \left(\pi q_{l}\left(\lambda^{\prime}\right)\right)$ (resp. $\left.g_{m}\left(\lambda^{\prime}\right)\right)$ since $\cos \left(\pi q_{l}\left(\lambda^{\prime}\right)\right)$ (resp. $\left.g_{m}\left(\lambda^{\prime}\right)\right)$ is not zero. Let $\mu_{1} \in D_{\gamma} \cap W$. We compare $b_{+}\left(G_{\lambda^{\prime}}\right)\left(\right.$ resp. $b_{+}\left(Q_{\lambda^{\prime}}\right)$ ) with $b_{+}\left(G_{\mu_{1}}\right)$ (resp. $\left.b_{+}\left(Q_{\mu_{1}}\right)\right)$. Since $b_{+}\left(G_{\mu_{1}}\right)=b_{+}\left(Q_{\mu_{1}}\right)$ by $\S 2.1$, to prove $b_{+}\left(G_{\lambda^{\prime}}\right)=b_{+}\left(Q_{\lambda^{\prime}}\right)$ we just have to show that if $\cos \left(\pi q_{k_{i}}\left(\mu_{1}\right)\right)>0$ for some $k_{i}, 1 \leq i \leq s$, then $g_{r_{\lambda^{\prime}}\left(k_{i}\right)}\left(\mu_{1}\right)>0$ and vice versa. Let us consider a small line segment with end points $\mu_{1}, \mu_{2}$ which passes from $D_{\gamma}$ to its neighbor $D_{\gamma^{\prime}}$, intersects the hyperplane $q_{k_{i}}(\mu)=q_{k_{i}}\left(\lambda^{\prime}\right)$ at $\mu_{0}$, and does not intersect any other hyperplanes. Then we have $\cos \left(\pi q_{k_{i}}\left(\mu_{0}\right)\right)=0$, so by lemma $1, g_{r_{\mu_{0}}\left(k_{i}\right)}\left(\mu_{0}\right)=0$. Again by lemma $1, r_{\mu_{0}}\left(k_{i}\right)$ depends only on $k_{i}$ and $q_{k_{i}}\left(\mu_{0}\right)=q_{k_{i}}\left(\lambda^{\prime}\right)$, so $r_{\mu_{0}}\left(k_{i}\right)=r_{\lambda^{\prime}}\left(k_{i}\right)$. As $\mu$ goes from $\mu_{1}$ to $\mu_{2}$ on the above line segment, $\cos \left(\pi q_{k_{i}}(\mu)\right), g_{r_{\lambda^{\prime}}\left(k_{i}\right)}(\mu)$ change their signs while the signs of all other $\cos \left(\pi q_{i}(\mu)\right), g_{j}(\mu)$ 's do not change. By $\S 2.1, b_{+}\left(Q_{\mu_{l}}\right)=b_{+}\left(Q_{\mu_{l}}\right), l=1,2$, it follows that if $\cos \left(\pi q_{k_{i}}\left(\mu_{1}\right)\right)>0$ for some $k_{i}, 1 \leq i \leq s$, then $g_{r_{\lambda^{\prime}}\left(k_{i}\right)}\left(\mu_{1}\right)>0$ and vice versa. So we have proved that $b_{+}\left(G_{\lambda^{\prime}}\right)=b_{+}\left(Q_{\lambda^{\prime}}\right)$, and since $b_{0}\left(G_{\lambda^{\prime}}\right)=b_{0}\left(Q_{\lambda^{\prime}}\right)$ by lemma 1 , and both $G_{\lambda^{\prime}}$ and $Q_{\lambda^{\prime}}$ have $N$ elements, theorem 1 is proved for $\lambda^{\prime} \in D$ which lies on the boundary of some $D_{\gamma}$.

By $§ 2.1, \S 2.2$, theorem 1 is proved.

2.3. Zuber's conjecture. To describe Zuber's conjecture, we have to introduce some notations from [Z1] to which the reader is referred for more details.

Let $\Lambda_{1}, \ldots, \Lambda_{N-1}$ be the fundamental weights of $S L(N)$. Let $k \in \mathbb{N}$. Recall that the set of integrable weights of the affine algebra $\widehat{S L(N)}$ at level $k$ is the following subset of the weight lattice of $S L(N)$ :

$$
P_{++}^{(h)}=\left\{\lambda=\lambda_{1} \Lambda_{1}+\ldots+\lambda_{N-1} \Lambda_{N-1} \mid \lambda_{i} \in \mathbb{N}, \lambda_{1}+\ldots+\lambda_{N-1}<h\right\}
$$


where $h=k+N$. This set admits a $\mathbb{Z}_{N}$ automorphism generated by

$$
\sigma: \lambda=\left(\lambda_{1}, \lambda_{2}, \ldots, \lambda_{N-1}\right) \rightarrow \sigma(\lambda)=\left(h-\sum_{j=1}^{N-1} \lambda_{j}, \lambda_{1}, \ldots, \lambda_{N-2}\right)
$$

. We then introduce the weights $e_{i}$ of the standard $N$-dimensional representation of $S L(N)$

$$
e_{1}=\Lambda_{1}, e_{i}=\Lambda_{i}-\Lambda_{i-1}, i=2, \ldots, N-1, e_{N}=-\lambda_{N-1}
$$

endowed with the scalar product $\left(e_{i}, e_{j}\right)=\delta_{i j}-\frac{1}{N}$. We shall be concerned with type II class of graphs introduced in section 1 of [Z1]. These graphs generalize the classical $A, D, E$ Dynkin diagrams which may be regarded as related to the $S L(2)$ algebra. The axioms on these graphs are given in $\S 1.2$ of [Z1] as follows:

(1) A set $\nu$ of $|\nu|=n$ vertices is given. These vertices are denoted by Latin letters $a, b, \ldots$. There exists an involution $a \rightarrow \bar{a}$ and the set $\nu$ admits a $\mathbb{Z}_{n}$ grading denoted by $\tau(a)$ such that $\tau(\bar{a})=-\tau(a) \bmod N$;

(2) A set of $N-1$ commuting $n \times n$ matrices $G_{p}, p=1,2, \ldots, N-1$ is given. Their matrix elements are assumed to be non-negative integers, so they may be regarded as adjacency matrices of $N-1$ graphs $g_{p} . g_{1}$ is also assumed to be connected;

(3) The edges of the graphs $g_{p}$ are compatible with the grading $\tau$ in the sense that $\left(G_{p}\right)_{a b}=0$ if $\tau(b) \neq \tau(a)+p \bmod N$;

(4) The matrices are transposed of one another $G_{p}^{t}=G_{N-p}$ and $\left(G_{p}\right)_{a b}=\left(G_{p}\right)_{\bar{b} \bar{a}}$;

(5) As a consequence of (2) and (4), the matrices $G_{p}$ are commuting normal matrices and may thus be simultaneously diagonalized in a common orthonormal basis. This basis, denoted by $\psi^{(\lambda, i)}$, is assumed to be labelled by the weights $\lambda$ of $S L(N)$, that are restricted to $P_{++}^{(h)}$, for some integer $h>N$, in a way that the eigenvalues $\gamma_{p}^{(\lambda)}$ have the form $\gamma_{p}^{(\lambda)}=\chi_{p}(M(\lambda))$, where $\chi_{p}$ is the ordinary character for the $p$-th fundamental representation of the group $S U(N)$, and $M(\lambda)$ denotes the diagonal matrix $M(\lambda)=\operatorname{diag}\left(\epsilon_{j}(\lambda)\right)_{j=1, \ldots, N}$. Here $\epsilon_{j}(\lambda):=\exp \left(-\frac{2 \pi i}{h}\left(e_{j}, \lambda\right)\right.$, and $i$ in $(\lambda, i)$ is an index integer, $1 \leq i \leq m_{\lambda}$ with $m_{\lambda}$ being the multiplicity of eigenvalue $\gamma_{p}^{(\lambda)}$. The set of $(\lambda, i)$ 's will be denoted by Exp.

There exists a special class of solutions known for all $N$ and $h>N$, namely the fusion graphs of the affine algebra $\widehat{S L(N)}$ at level $k=h-N$. The vertices are the integrable weights described above, i.e., $\nu=P_{++}^{(h)}$. The matrices $G_{p}$ are the Verlinde matrices, which describe the fusion by the $p$-th fundamental representation. The fusion rules are given on Page 288 of $[\mathrm{K}]$. Their diagonalization is known, thanks to the Verlinde formula (cf. Page 288 of $[\mathrm{K}]$ ), and the eigenvalues are the $\gamma_{p}^{(\lambda)}$, where $\lambda$ takes all the values in $=P_{++}^{(h)}$. We will call these graphs as regular graphs in this paper. In the case of $N=2$, these regular graphs reduce to the $A_{h-1}$ Dynkin diagrams.

More solutions are known (cf.[Z1]). In [X] (in particular Th.3.10 and (5) of Th.3.8), infinite series of such graphs are constructed from the maximal conformal 
inclusions of the form $S U(N) \subset G$ with $G$ being a simple and simply connected compact Lie group.

Given graphs of the previous type, let $V$ be a complex vector space with a basis $\alpha_{a}$ labelled by the vertices of the set $\nu$. A bilinear form $g$ is defined by :

$$
g_{a b}=\left\langle\alpha_{a}, \alpha_{b}\right\rangle=2 \delta_{a b}+G_{a b}
$$

in terms of the matrix $G_{a b}=\sum_{p=1}^{N-1}\left(G_{p}\right)_{a b} . g$ will be called the intersection form. This is the intersection form in the title of this paper.

The eigenvalue of the matrix $\left(g_{a b}\right)$ with eigenvector $\psi_{p}^{(\lambda, i)}$ is (cf. (34) of [Z1]):

$$
g^{(\lambda)}=\prod_{i=1}^{N}\left(1+\exp \left(-\frac{2 \pi i}{h}\left(e_{i}, \lambda\right)\right)\right)
$$

. For $(\lambda, i) \in \operatorname{Exp}$ define real numbers which depend only on $\lambda$ (cf. (46) of [Z1])by:

$$
q_{\lambda}^{(R)}:=\frac{1}{h} \sum_{j=1}^{N-1} j\left(\lambda_{j}-1\right)+\frac{(N-h)(N-1)}{2 h}
$$

- We can now state Zuber's conjecture on the signature of $g$ (cf. Conjecture 2.5 of $[\mathrm{Z1}])$ :

Zuber's Conjecture. The signature of the bilinear form $g$ for class II graphs is $(x+, y-, z 0)$ where $x$ is the number of $q_{\lambda}^{(R)}$ which fall in an interval $] 2 p-\frac{1}{2}, 2 p+\frac{1}{2}[$ for some $p \in \mathbb{Z}$ (p may depend on $\left.q_{\lambda}^{(R)}\right), y$ is the number of those in an interval ] $2 p^{\prime}+\frac{1}{2}, 2 p^{\prime}+\frac{3}{2}\left[\right.$ for some $p^{\prime} \in \mathbb{Z}\left(p^{\prime}\right.$ may depend on $\left.q_{\lambda}^{(R)}\right)$, and $t=n-r-s$ is the number of those $q_{\lambda}^{(R)}$ which are half-integers.

We now prove this conjecture.

Let us first notice a simple consequence of the axioms on the graphs. It follows from Prop.1.2 of [Z1] that Exp is invariant under the action of $\sigma$. In fact, if $\sum_{a} \psi_{a}^{(\lambda, i)} a$ is an eigenvector of $G_{p}$ with eigenvalue $\gamma_{p}^{(\lambda)}$, then Prop.1.2 of [Z1] implies that $\sum_{a} \psi_{a}^{(\lambda, i)} \exp \left(\frac{2 \pi i \tau(a)}{N}\right) a$ is an eigenvector of $G_{p}$ with eigenvalue $\gamma_{p}^{(\sigma(\lambda))}$. Since $a \rightarrow \exp \left(\frac{2 \pi i \tau(a)}{N}\right) a$ is an invertible map, it follows that the multiplicity of eigenvalue $\gamma_{p}^{(\sigma(\lambda))}$ is the same as that of eigenvalue $\gamma_{p}^{(\lambda)}$. We can therefore define $\sigma(\lambda, i)=(\sigma(\lambda), i)$. It follows that Exp can be written as a disjoint union of the orbits under the action of $\sigma$. To prove Zuber's conjecture, we just have to show it is true on each orbit.

Let $(\lambda, i) \in \operatorname{Exp}$ and let $d$ be the smallest positive integer such that $\sigma^{d}(\lambda)=$ $\lambda$. Then $d \mid N$ and let $N=d d_{1}$. Let $G_{\lambda}^{\prime}:=\left\{g^{\left(\sigma^{i}(\mu)\right)}, i=1,2, \ldots, d\right\}, Q_{\lambda}^{\prime}:=$ $\left\{\cos \left(\pi q_{\sigma^{i}(\mu)}^{(R)}\right), i=1,2, \ldots, d\right\}$, we need to show

$$
b_{+}\left(G_{\lambda}^{\prime}\right)=b_{+}\left(Q_{\lambda}^{\prime}\right), b_{0}\left(G_{\lambda}^{\prime}\right)=b_{0}\left(Q_{\lambda}^{\prime}\right), b_{-}\left(G_{\lambda}^{\prime}\right)=b_{-}\left(Q_{\lambda}^{\prime}\right) .
$$


Note that $b_{0}\left(G_{\lambda}^{\prime}\right)=b_{0}\left(Q_{\lambda}^{\prime}\right)$ was already noticed on page 17 of [Z1]. Let $\lambda^{\prime}=$ $\left(\frac{\lambda_{1}}{h}, \ldots, \frac{\lambda_{N-1}}{h}\right)$, then $p_{i}\left(\lambda^{\prime}\right)=\frac{\left(e_{i}, \lambda\right)}{h}, i=1,2, \ldots, N$. To use theorem 1 , we make use of the following identities which follow from the definitions:

$$
q_{\sigma^{-j}(\lambda)}^{(R)}=q_{j}\left(\lambda^{\prime}\right)
$$

and

$$
\begin{aligned}
g^{\left(\sigma^{j}(\lambda)\right)} & =\prod_{l=1}^{N}\left(1+\exp \left(+\frac{2 \pi i j}{N} \epsilon_{l}(\lambda)\right)\right) \\
& =\prod_{l=1}^{N} 2 \cos \left(\pi\left(p_{l}\left(\lambda^{\prime}\right)-\frac{j}{N}\right)\right) \times \exp \left(\pi i j-\sum_{l=1}^{N} \frac{1}{h}\left(\lambda, e_{l}\right)\right) \\
& =(-1)^{j} \prod_{l=1}^{N} 2 \cos \left(\pi\left(p_{l}\left(\lambda^{\prime}\right)-\frac{j}{N}\right)\right) \\
& =g_{j}\left(\lambda^{\prime}\right)
\end{aligned}
$$

- Now it is clear that the size of $G_{\lambda^{\prime}}$ (resp. $Q_{\lambda^{\prime}}$ ) is $d_{1}$ times the size of $G_{\lambda}^{\prime}$ (resp. $\left.Q_{\lambda}^{\prime}\right)$ and we have:

$$
b_{+}\left(G_{\lambda^{\prime}}\right)=d_{1} b_{+}\left(G_{\lambda}^{\prime}\right), b_{0}\left(G_{\lambda^{\prime}}\right)=d_{1} b_{0}\left(G_{\lambda}^{\prime}\right), b_{-}\left(G_{\lambda^{\prime}}\right)=d_{1} b_{-}\left(G_{\lambda}^{\prime}\right)
$$

and

$$
b_{+}\left(Q_{\lambda^{\prime}}\right)=d_{1} b_{+}\left(Q_{\lambda}^{\prime}\right), b_{0}\left(Q_{\lambda^{\prime}}\right)=d_{1} b_{0}\left(Q_{\lambda}^{\prime}\right), b_{-}\left(Q_{\lambda^{\prime}}\right)=d_{1} b_{-}\left(Q_{\lambda}^{\prime}\right)
$$

. By theorem 1, we have proved:

$$
b_{+}\left(G_{\lambda}^{\prime}\right)=b_{+}\left(Q_{\lambda}^{\prime}\right), b_{0}\left(G_{\lambda}^{\prime}\right)=b_{0}\left(Q_{\lambda}^{\prime}\right), b_{-}\left(G_{\lambda}^{\prime}\right)=b_{-}\left(Q_{\lambda}^{\prime}\right)
$$

Let us summarize the result in the following:

Corollary 1. Zuber's Conjecture as stated above is true.

\section{§5. Conclusions And Questions}

In this paper we proved Zuber's conjecture on the signature of certain intersection forms by using theorem 1 .

Our results imply that the infinite series of graphs which are constructed in $[\mathrm{X}]$ by using subfactors associated with conformal inclusions satisfy Zuber's conjecture. This lends further support to the idea that these graphs may be associated with the integrable models in [CV] which is the basis of Zuber's conjecture. Such a relation is not very clear and should be very interesting. 


\section{REFERENCES}

[CV] S.Cecotti and C.Vafa, Nucl.Phys. 367 (1991) 359-461; Comm.Math.Phys. 158 (1993) 569-644.

[K] V.Kac, Infinite dimensional Lie algebras,third edition, Cambridge University Press.

[S] J.Steenbrink, Composito Math. 34(1977) 211-223.

[GZV] S.M. Gusein-Zade and A. Varchenko, Selecta Math. (N.S.) 3(1997), no.1, 79-97.

[X] F. Xu, New braided endomorphisms from conformal inclusions, Comm. Math. Phys., (1998) (in press).

[Z1] J.-B. Zuber, Generalized Dynkin diagrams and root systems and their folding , hep-th 9707046, to appear in the proceedings of the Taniguchi Symposium Topological Field theory, Primitive forms and Related Topics, Kyoto Dec. 1996, M. Kashiwara, A. Matsuo, K. Saito and I. Satake eds, Birkhaüser.

[Z2] J.-B. Zuber, Comm.Math.Phys. 179 (1996) 265-294. 